\title{
Submerge Detection Of Sensor Nodes
}

\section{S.Surya ${ }^{1}$, Berlin Mary ${ }^{2}$, Christo Ananth ${ }^{3}$}

P.G.Scholars, Department of CSE,Francis Xavier Engineering College, Tirunelveli ${ }^{1,2}$ Assistant professor, Department of ECE, Francis Xavier Engineering College, Tirunelveli ${ }^{3}$

\section{Abstract}

In this paper, Underwater networking sensor nodes provide the oceanographic collection of data and monitoring of unmanned or autonomous underwater vehicle to explore sea recourses and gathering of scientific data. The sensor network contains the statistical data about the sensor nodes. High speed optical communication is provided between the nodes in a point to point fashion. The design emphasis on the modulation and demodulation of the signals and thereby providing the synchronization between the nodes. The challenges include waterproofing, casing, calibration. Furthermore the research issues are outlined.

\section{INTRODUCTION:}

In underwater networking, the oceanographic collection of data users radio meters to estimate the sea surface temperature, scatter meters to measure the wave disturbances, wind speeds and directions and altimeters to measure ocean surface deformation to determine sea surface slopes and ocean current. The underwater vehicles are classified into autonomous underwater vehicle(AUV), to detect and map the underwater survey machines such as a hazards. It is also determined as $\operatorname{UUV}($ Unmanned under water vehicles), also known as underwater drones. These are the vehicles that operate in underwater without a human occupant. It would constitute a kind of a

amplify analog signals. Active sensor actively probe the environment.

The above described features enable wide range of applications for the underwater sensor networks:

- Ocean morsel networks: The sampling of the ocean deals with the exhaustive factors which are determined through the sensors. The robotic vehicles are used to sample the models in the ocean. The characteristics of the ocean are observed and predict the activities that are 
detected through the sensor nodes in the network. The sensor network are responsible for the varieties of the functionalities like the pollution, exploration and disaster in the ocean.

- Monitoring the stomp ground: The monitoring of the stomp ground includes the monitoring of the chemical, biological and nuclear pollution that occurs in the underwater. It may give the details about the amount of chemicals that are mixed with the water. It may derive the serious factors that disturbs the organisms in the water. It also monitors the oceanic currents, climatic changes and weather forecasting. When there are periodic changes in the high and low tides is very, which helps to derive the possibility of the disasters that may occur.

- Sunken explorations: It is very much important to determine the oilfields and reservoirs in the underwater. It helps in the building in the sensor networks in the underwater.In the exploration it may also include the findings of the minerals. The underwater provides wide variety of the ores and minerals that are very essential for the human beings.
- Holocaust exploration: In the exploration of the holocaust, there is a greatest possibility to determine the disasters before it occurs. It also provides the measures of the events to be occurred in the mere future from the remote areas. The submarine earthquakes can be predicted as much as earlier to avoid the damages in the coastal areas.

- Aided triangulation: The assist of the sensors is done to identify the hazards on the ocean. It helps in locating the dangerous rocks in the seabed and the shoals in the shallow waters. The submerging of the areas are assisted to determine that there are defected areas in the coastal region thereby providing an improvement in the affected areas which also affect the micro organisms in the ocean.

- Distributed shield shadowing: The shadowing is the mechanism to monitor the areas.It helps to target any particular area through which the situation in any coastal area can be determined.It enhances on the property of providing shield that is, the detection of the intrusion are done such that all the events are done periodically without any problem that leads to the death of the living organisms.The sensory nodes provides the highest accuracy in 
the gathering of the information and exploration of the underwater resources efficiently.

- Mine reconnoiter: All the operations are performed concurrently through the sensors that are used for assessing the stomp ground thereby detecting the mine-like objects for the discovery of the minerals.

Since the world war II, the findings in the submerge is unexplored. At the earlier stage, radio waves were transmitted at the lower frequencies of about $30-300 \mathrm{~Hz}$.At the bottom layer of the ocean if there is any difficulties exists then it is difficult to overcome them. The disadvantages are as follows:

- $\quad$ No concurrent monitoring: the beginning of the monitoring mission, the recorded data can not be accessed until the instruments are recovered.

- No disaster detection: If any failure occurs, it is not detected before the instruments are recovered.

- Limited Storage: only limited data can be stored on the sensors. The onboard storage devices are memories and hard disk.

The design includes several challenges, they are as follows:
- The limitations in the bandwidth

- The limitation of the power in the battery that cannot be recharged.

- Failure arises due to corrosion and fouling.

- Sometimes shadow zones causes the disconnection.

- Delay in the propagation in the underwater.

\section{Communications and working}

\section{technologies of the sensor networks}

In this section we discuss about how the sensor node communicate with each other in the underwater. It relates a various technologies and terminologies to handle the sensor node that they communicate with each other without any intrusion. also, we discuss about the various applications, tools and methods.

- Physical layer: Multi-path propagation creates signal echoes that arrive with varying delays. Delay spreading depends on the system location, and can range from a few milliseconds to several hundreds of milliseconds. In a wideband system, this leads to a frequency selective channel transfer function as different 
April 17, 2015

frequency components may exhibit substantially different attenuation. The channel response and the instantaneous power often exhibit smallscale, fast variations, typically caused by scattering and the rapid motion of the sea surface (waves) or of the system itself. While large-scale variations influence power control at the transmitter, small-scale variations influence the design of adaptive signal processing algorithms at the receiver.

The characteristics of the physical layer influence medium access and higher layer protocol design. For example, the same network protocol may perform differently under a different frequency allocation-moving to a higher frequency region will cause more attenuation to the desired signal, but the interference will attenuate more as well, possibly boosting the overall performance. Also, propagation delay and packet duration matter, since a channel that is sensed to be free may nonetheless contain interfering packets; their length will affect the probability of collisions and the efficiency of re-transmission (throughput).Finally, power control, coupled with intelligent routing, can greatly help us to limit interference.
- Medium access control and resource

sharing: In designing resource-sharing schemes for underwater networks, one needs to keep in mind the peculiar characteristics of the acoustic channel. Most relevant in this context are long delays, frequency-dependent attenuation and the relatively long reach of acoustic signals. In addition, the bandwidth constraints of acoustic hardware (and the transducer in particular) must also be considered.

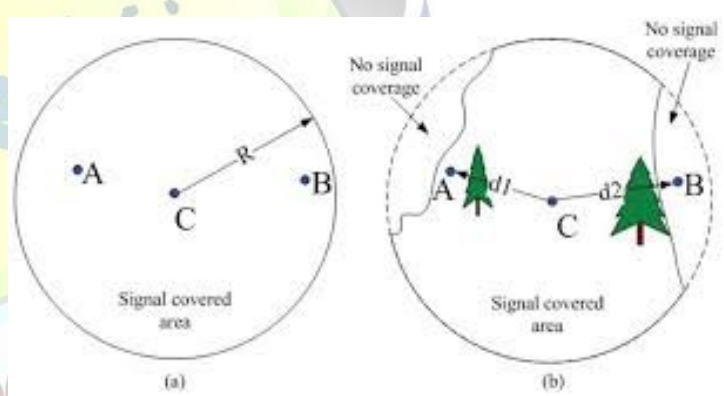

The network layer, routing and transport: In large networks, it is unlikely that any pair of nodes can communicate directly, and multi-hop operation, by which intermediate nodes are used to forward messages towards the final destination, is typically used. routing protocols are used to determine a variable route that a packet should follow through a topology. The design of transport protocols in underwater acoustic networks is another critical issue. Protocols such as TCP are designed for low to 
moderate latencies, not the large fractions of a second commonly encountered in underwater networks, and limited bandwidth and high loss suggest that end-to-end retransmission will perform poorly.

Finally, an important issue is that of topology control, where nodes sleep to reduce energy while maintaining network connectivity. Although coordination and scheduling mechanisms can be used for this purpose.

\section{- Sensing and application techniques:}

Traditional biology and oceanography rely on samples that are taken in the environment and returned to the laboratory for analysis. As traditional underwater research has assumed personnel on site, the cost of sample return is relatively small compared with the cost of getting the scientist to the site. With lower cost sensor networks and AUVs, we expect the costs of sample-return relative to in situ sensing to force revisiting these assumptions. Algorithms for managing underwater sensing, sensor fusion, and coordinated and adaptive sensing are just beginning to develop. As the field matures, we look forward to work involving online, adaptive sampling using communicating AUVs. Some types of underwater sensors are easy and inexpensive, but many rapidly become difficult and expensive- from a few dollars to thousands or more. Inexpensive sensors include pressure sensing, which can give approximate depth, and photo-diodes and thermistors that measure ambientlight and temperature.

Simulators and models: Wireless communication channel simulation, which is OPNET platform built-in, adopts 14 end- to-end pipeline stage (Pipeline Stage) to simulate the transmission of data frames in the channel as truly as possible, and provides a default model for each pipeline stage However, OPNET pipeline stage model just simulates the air wireless channel, and is not suitable for the underwater acoustic channel. Therefore, we need to improve existing models to the underwater acoustic channel. generally, the speed of wind is set as $10 \mathrm{~m} / \mathrm{s}$, and the waters shipping density is set as 0.6. The network includes a source node (node 0), a destination node(node 2) and two relay nodes (node 1 , node 3). The depth and locomotion speed of the nodes are set as: node 2 (underwater $100 \mathrm{~m}$, $30 \mathrm{~km} / \mathrm{h}$ ), node 1 (underwater $50 \mathrm{~m}$, 
$11 \mathrm{~km} / \mathrm{h}$ ), node 3 (underwater $60 \mathrm{~m}, 11 \mathrm{~km} / \mathrm{h}$ ), node 0 (in the surface, $10 \mathrm{~m} / \mathrm{h}$ ).

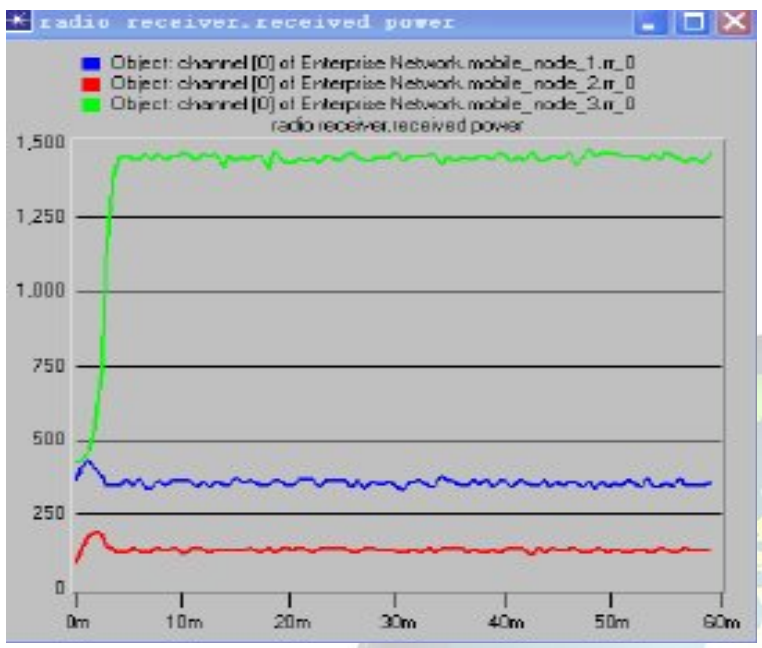

Fig:The received power curve of node1,node2 and node 3

\section{3.conclusions and future challenges}

This paper we describe the challenges posed in the underwater with reference to monitoring the applications in the ocean environment. The ultimate objective of the paper is to encourage more researchers for the development of advanced communication techniques for the efficient communication and networking. In addition ,integration and testing of current ideas will stress the seams that are often hidden in the research, such as total system cost, energy requirements and robustness. The objectives were to determined the minimum number of sensor to be deployed for the efficient communications, thereby studying the robustness to determine the failure of nodes, and estimate the number of required redundant sensors.

\section{References}

1.Ian F. Akyildiz, Dario Pompili, Tommaso Melodia. Challenges for EfficientCommunication inUnderwater Acoustic Sensor Networks,January 2005.

2. I. Vasilescu, K. Kotay, and D. Rus, M. Dunbabin and P. Corke, Data Collection, Storage, and Retrieval with an Underwater Sensor Network,april2004

3. John Heidemann, Wei Ye, Jack Wills, Affan Syed, Yuan Li Information Sciences Institute, University of Southern California, Research Challenges and Applications for Underwater Sensor Networking,July 2005

4. Chengsheng Pan1;2, Liangchen Jia1;3, Ruiyan Cail;3 and Yuanming Ding1;3, modeling and simulation of channel for underwater communication network, International Journal ofInnovative Computing, Information and Control Volume 8, Number 3(B), March 2012.

5. John Heidemann, Milica Stojanovic and Michele Zorzi, advances and challenges Underwater sensor networks: applications,June 2012

6. Raja Jurdak, Antonio G. Ruzzelli, Gregory M.P. O'Hare, Design Considerations for 
Deploying Underwater Sensor Networks,July 2010

7. B. Chen, K. Jamieson, H. Balakrishnan, and R. Morris. Span: an energyefficient coordination algorithm for topology maintenance in ad hocwireless networks. In Proceedings of the ACM International Conferenceon Mobile Computing and Networking, Rome, Italy, July 2001.

8. N. B. Priyantha, A. Chakraborty, and H. Balakrishnan. The cricket location-support system. In Proceedings of the 6th ACM International, Conference on Mobile Computing and Networking, pages 32-43, Boston, MA, USA, Aug. 2000.

9.L. Freitag and M. Stojanovic, "Acoustic communications for regional undersea observatories," in Proceedings of Oceanology International, London, U.K., mar 2002.

10.W. Ye, J. Heidemann, and D. Estrin, "An energy-efficient MAC protocol for wireless sensor networks," in Proceedings of IEEE INFOCOM 2002, vol. 3, New York, NY, USA, June, pp. 1567-1576.

11.Y. Sankarasubramaniam, O. B. Akan, and I. F. Akyildiz, "ESRT: eventto- sink reliable transport for wireless sensor networks," in Proceedings of ACM MOBIHOC 2003, Annapolis, MD, USA, June, pp. 177-188.

12.Autonomous Ocean Sampling Network (AOSN)II,collaborative project.

http://www.princeton.edu/ dcsl/aosn/. 